\title{
ON AN APPROXIMATION ORDER OF THE OPTIMAL STOPPING PROBLEM FOR $n$-DIMENSIONAL DIFFUSION PROCESSES
}

\author{
GIORGI LOMINASHVILI
}

\begin{abstract}
An approximation order of the optimal stopping problem for multidimensional diffusion processes by the corresponding semidiscretization is considered.
\end{abstract}

2000 Mathematics Subject Classification: 60G40.

Key words and phrases: Optimal stopping, $n$-dimensional diffusion process, approximation order.

\section{Preliminaries}

Let $(\Omega, \mathcal{F}, P)$ be a probability space, and $W_{t}=\left(W_{t}^{1}, \ldots, W_{t}^{n}\right)$ be $n$-dimensional standard Brownian motion defined on this space. Denote by $F^{W}=$ $\left(\mathcal{F}_{t}^{W}\right)_{t \geq 0}$ the natural filtration of $W_{t}$ completed with respect to $P$.

On the time interval $T=[0, \infty)$ let us consider the stochastic differential equation

$$
d X_{s}=b\left(X_{s}\right) d s+\sigma\left(X_{s}\right) d W_{s}, \quad X_{0}=x \in \mathbb{R}^{n},
$$

where

A. $b(x)=\left(b^{1}(x), \ldots, b^{n}(x)\right)$ and $\sigma(x)=\left(\sigma_{j}^{i}(x)\right), i, j=1, \ldots, n$, are locally Lipschitz functions from $\mathbb{R}^{n}$ into $\mathbb{R}^{n}$ and the space of $n \times n$ real matrices, respectively, which satisfy the linear growth condition

$$
\|b(x)\| \leq k(1+\|x\|), \quad\|\sigma(x)\| \leq k(1+\|x\|)
$$

with a constant $k$; here $\|\cdot\|$ stands for the Euclidean norm (Frobenius norm for $\sigma(x))$.

As is well known (see [2], Ch. VIII, $\S 2$, Theorem 4), under condition A the stochastic differential equation (1) has a unique strong solution $X_{t}=$ $\left(X_{t}^{1}, \ldots, X_{t}^{n}\right)$ which is a Markov diffusion process.

Let $D$ be a bounded domain in the space $\mathbb{R}^{n}$, and $\bar{D}$ and $\partial D$ be its closure and smooth boundary (say, of $C^{2}$ ), respectively. Assume that $g=g(x)$ is a Lipschitz function defined on $\bar{D}$ with the Lipschitz constant $L$, and $c=c(x)$ is a continuous function defined on $\bar{D}$.

Let us introduce the first moment at which the process $X_{t}, t \in T$, exits from the domain $D$ :

$$
\sigma(D)=\inf \left(t \geq 0: X_{t} \notin D\right)
$$


for which $\sup _{x \in \bar{D}} E_{x} \sigma(D)<\infty$ and let us consider the optimal stopping problem in the domain $\bar{D}$ :

$$
S(x)=\sup _{\tau \in \mathfrak{M}} E_{x}\left[g\left(X_{\tau \wedge \sigma(D)}\right) e^{-r(\tau \wedge \sigma(D))}+\int_{0}^{\tau \wedge \sigma(D)} c\left(X_{t}\right) e^{-r t} d t\right],
$$

where $P_{x}$ is the probability measure corresponding to the initial condition $X_{0}=$ $x, E_{x}$ is the sign of taking expectation w.r.t. $P_{x}, \mathfrak{M}$ is the class of all stopping moments w.r.t. the filtration $F^{W}=\left(\mathcal{F}_{t}^{W}\right)_{t \geq 0}$, and $r>0$ is some constant.

Let $X_{t}^{\sigma(D)}=X_{t \wedge \sigma(D)}$ be the stopped process which is a solution of the following stochastic differential equation:

$$
d X_{s}^{\sigma(D)}=\widetilde{b}\left(X_{s}^{\sigma(D)}\right) d s+\widetilde{\sigma}\left(X_{s}^{\sigma(D)}\right) d W_{s},
$$

where $\tilde{b}(x)=b(x) \chi_{D}(x), \tilde{\sigma}(x)=\sigma(x) \chi_{D}(x)$ and $\chi_{D}(x)$ is the characteristic function of $D$.

The optimal stopping problem (2) can be equivalently rewritten in terms of stopped diffusion processes as

$$
S(x)=\sup _{\tau \in \mathfrak{M}} E_{x}\left[g\left(X_{\tau}^{\sigma(D)}\right) e^{-\int_{0}^{\tau} \widetilde{r}\left(X_{s}^{\sigma(D)}\right) d s}+\int_{0}^{\tau} \widetilde{c}\left(X_{t}^{\sigma(D)}\right) e^{-\int_{0}^{t} \widetilde{r}\left(X_{s}^{\sigma(D)}\right) d s} d t\right],
$$

where $\widetilde{r}(x)=r \cdot \chi_{D}(x), \widetilde{c}(x)=c(x) \cdot \chi_{D}(x)$.

Assume that $h, 0<h<1$, is the parameter that tends to zero. Denote by $\mathfrak{M}_{h}$ the class of stopping moments $\tau_{h}$, where $\tau_{h}$ takes the values $0, h, \ldots, n h, \ldots$, and the set $\left\{\tau_{h} \leq n h\right\} \in \mathcal{F}_{n h}^{W}$.

The discrete analog of the problem (3) is written as follows (see [1]):

$$
S_{h}(x)=\sup _{\tau_{h} \in \mathfrak{M}_{h}} E_{x}\left[g\left(X_{\tau_{h}}^{\sigma(D)}\right) e^{-\int_{0}^{\tau_{h}} \widetilde{r}\left(X_{s}^{\sigma(D)}\right) d s}+\int_{0}^{\tau_{h}} \widetilde{c}\left(X_{t}^{\sigma(D)}\right) e^{-\int_{0}^{t} \widetilde{r}\left(X_{s}^{\sigma(D)}\right) d s} d t\right] .
$$

Denote by $\tau^{*}$ the optimal stopping time of problem (3) (see [3], Ch. 3, Theorem 3). Besides, introduce the so-called pseudo-optimal stopping moment $\tau_{h}^{\prime}$ defined as follows:

$$
\tau_{h}^{\prime}=\inf \left(n h: n h \geq \tau^{*}\right)
$$

which is an admissible stoping time from $\mathfrak{M}_{h}$. In [1], requiring only continuity of $g(x)$, Bensoussan and Robins showed that $S_{h}(x)$ converges to $S(x)$ as $h \rightarrow 0$.

\section{Formulation and Proof of the Basic Result}

Theorem. Let $\left(X_{t}, P_{x}\right), t \in T$, be an $n$-dimensional diffusion process given by the stochastic differential equation (1) with the coefficients $b(x)$ and $\sigma(x)$ satisfying condition $\mathbf{A}$, and $g(x)$ be the Lipschitz function defined on $\bar{D}$. Then for the functions $S(x)$ and $S_{h}(x)$ defined by (3) and (4), respectively, the following estimate is valid:

$$
\sup _{x \in \bar{D}}\left|S(x)-S_{h}(x)\right| \leq p \cdot h^{1 / 2}
$$


where $p=C_{1}+r H+L \sqrt{2 n C_{2}^{2}+2 n^{2} C_{3}^{2}}, r$ is introduced in relation (2) and $H$, $C_{1}, C_{2}, C_{3}$ are the constants such that for $x \in \bar{D}$

$$
|g(x)| \leq H, \quad|c(x)| \leq C_{1}, \quad\left|b^{i}(x)\right| \leq C_{2}, \quad\left|\sigma_{j}^{i}(x)\right| \leq C_{3}, \quad i, j=1, \ldots, n .
$$

Proof. It is obvious from (4) that

$$
S_{h}(x) \geq E_{x}\left[g\left(X_{\tau_{h}^{\prime}}^{\sigma(D)}\right) e^{-\int_{0}^{\tau_{h}^{\prime}} \widetilde{r}\left(X_{s}^{\sigma(D)}\right) d s}+\int_{0}^{\tau_{h}^{\prime}} \widetilde{c}\left(X_{t}^{\sigma(D)}\right) e^{-\int_{0}^{t} \widetilde{r}\left(X_{s}^{\sigma(D)}\right) d s} d t\right],
$$

and therefore

$$
\begin{aligned}
0 \leq & S(x)-S_{h}(x) \leq E_{x}\left[g\left(X_{\tau^{*}}^{\sigma(D)}\right) e^{-\int_{0}^{\tau^{*}} \widetilde{r}\left(X_{s}^{\sigma(D)}\right) d s}+\int_{0}^{\tau^{*}} \widetilde{c}\left(X_{t}^{\sigma(D)}\right) e^{-\int_{0}^{t} \widetilde{r}\left(X_{s}^{\sigma(D)}\right) d s} d t\right] \\
& -E_{x}\left[g\left(X_{\tau_{h}^{\prime}}^{\sigma(D)}\right) e^{-\int_{0}^{\tau_{h}^{\prime}} \widetilde{r}\left(X_{s}^{\sigma(D)}\right) d s}+\int_{0}^{\tau_{h}^{\prime}} \widetilde{c}\left(X_{t}^{\sigma(D)}\right) e^{-\int_{0}^{t} \widetilde{r}\left(X_{s}^{\sigma(D)}\right) d s} d t\right]
\end{aligned}
$$

Clearly,

$$
\begin{aligned}
& g\left(X_{\tau^{*}}^{\sigma(D)}\right) e^{-\int_{0}^{\tau^{*}} \widetilde{r}\left(X_{s}^{\sigma(D)}\right) d s}-g\left(X_{\tau_{h}^{\prime}}^{\sigma(D)}\right) e^{-\int_{0}^{\tau_{h}^{\prime}} \widetilde{r}\left(X_{s}^{\sigma(D)}\right) d s} \\
& =\left(g\left(X_{\tau^{*}}^{\sigma(D)}\right)-g\left(X_{\tau_{h}^{\prime}}^{\sigma(D)}\right)\right) e^{-\int_{0}^{\tau_{h}^{\prime}} \widetilde{r}\left(X_{s}^{\sigma(D)}\right) d s} \\
& \quad+g\left(X_{\tau^{*}}^{\sigma(D)}\right) e^{-\int_{0}^{\tau^{*}} \widetilde{r}\left(X_{s}^{\sigma(D)}\right) d s}\left(1-e^{-\int_{\tau^{*}}^{\tau_{h}^{\prime}} \widetilde{r}\left(X_{s}^{\sigma(D)}\right) d s}\right) .
\end{aligned}
$$

If we use the elementary inequality $1-e^{-z}<z, z>0$, then (6) leads to the following inequality

$$
\begin{aligned}
& g\left(X_{\tau^{*}}^{\sigma(D)}\right) e^{-\int_{0}^{\tau_{0}^{*}} \widetilde{r}\left(X_{s}^{\sigma(D)}\right) d s}-g\left(X_{\tau_{h}^{\prime}}^{\sigma(D)}\right) e^{-\int_{0}^{\tau_{h}^{\prime}} \widetilde{r}\left(X_{s}^{\sigma(D)}\right) d s} \\
& \quad \leq\left|g\left(X_{\tau^{*}}^{\sigma(D)}\right)-g\left(X_{\tau_{h}^{\prime}}^{\sigma(D)}\right)\right|+\left|g\left(X_{\tau^{*}}^{\sigma(D)}\right)\right| \int_{\tau^{*}}^{\tau_{h}^{\prime}} \widetilde{r}\left(X_{s}^{\sigma(D)}\right) d s .
\end{aligned}
$$

Taking into account (7) in (5), we obtain

$$
\begin{gathered}
0 \leq S(x)-S_{h}(x) \leq E_{x}\left[\left|g\left(X_{\tau^{*}}^{\sigma(D)}\right)-g\left(X_{\tau_{h}^{\prime}}^{\sigma(D)}\right)\right|+\left|g\left(X_{\tau^{*}}^{\sigma(D)}\right)\right| \int_{\tau^{*}}^{\tau_{h}^{\prime}} \widetilde{r}\left(X_{s}^{\sigma(D)}\right) d s+\right. \\
\left.+\int_{\tau^{*}}^{\tau_{h}^{\prime}}\left|\widetilde{c}\left(X_{t}^{\sigma(D)}\right)\right| e^{-\int_{0}^{t} \widetilde{r}\left(X_{s}^{\sigma(D)}\right) d s} d t\right] .
\end{gathered}
$$


We have

$$
\begin{gathered}
\int_{\tau^{*}}^{\tau_{h}^{\prime}}\left|\widetilde{c}\left(X_{t}^{\sigma(D)}\right)\right| e^{-\int_{0}^{t} \widetilde{r}\left(X_{s}^{\sigma(D)}\right) d s} d t \leq C_{1}\left(\tau_{h}^{\prime}-\tau^{*}\right) \leq C_{1} h, \\
\left|g\left(X_{\tau^{*}}^{\sigma(D)}\right)\right| \int_{\tau^{*}}^{\tau_{h}^{\prime}} \widetilde{r}\left(X_{s}^{\sigma(D)}\right) d s \leq r H\left(\tau_{h}^{\prime}-\tau^{*}\right) \leq r H h .
\end{gathered}
$$

It remains to estimate the value $E_{x}\left|g\left(X_{\tau^{*}}^{\sigma(D)}\right)-g\left(X_{\tau_{h}^{\prime}}^{\sigma(D)}\right)\right|$. Since $g(x)$ is the Lipschitz function defined on $\bar{D}$, we have

$$
\begin{gathered}
E_{x}\left|g\left(X_{\tau^{*}}^{\sigma(D)}\right)-g\left(X_{\tau_{h}^{\prime}}^{\sigma(D)}\right)\right| \leq L E_{x}\left\|X_{\tau^{*}}^{\sigma(D)}-X_{\tau_{h}^{\prime}}^{\sigma(D)}\right\| \leq L\left(E_{x}\left\|X_{\tau^{*}}^{\sigma(D)}-X_{\tau_{h}^{\prime}}^{\sigma(D)}\right\|^{2}\right)^{1 / 2}, \\
E_{x}\left\|X_{\tau^{*}}^{\sigma(D)}-X_{\tau_{h}^{\prime}}^{\sigma(D)}\right\|^{2}=E_{x}\left\|\int_{\tau^{*}}^{\tau_{h^{\prime}}} \widetilde{b}\left(X_{t}^{\sigma(D)}\right) d t+\int_{\tau^{*}}^{\tau_{h^{\prime}}} \widetilde{\sigma}\left(X_{t}^{\sigma(D)}\right) d W_{t}\right\|^{2} \\
\leq 2 E_{x} \sum_{i=1}^{n}\left[\int_{\tau^{*}}^{\tau_{h^{\prime}}} \widetilde{b}^{i}\left(X_{t}^{\sigma(D)}\right) d t\right]^{2}+2 E_{x} \sum_{i=1}^{n}\left[\int_{\tau^{*}}^{\tau_{h^{\prime}}} \sum_{j=1}^{n}\left(\widetilde{\sigma}_{j}^{i}\left(X_{t}^{\sigma(D)}\right)\right)^{2} d t\right] \\
\leq E_{x}\left[2 n C_{2}^{2}\left(\tau_{h}^{\prime}-\tau^{*}\right)^{2}+2 n^{2} C_{3}^{2}\left(\tau_{h}^{\prime}-\tau^{*}\right)\right] .
\end{gathered}
$$

Finally,

$$
\sup _{x \in \bar{D}}\left|S(x)-S_{h}(x)\right| \leq C_{1} h+r H h+\sqrt{h\left(2 n C_{2}^{2}+2 n^{2} C_{3}^{2}\right)} \cdot L \leq p \cdot h^{1 / 2} .
$$

\section{REFERENCES}

1. A. Bensoussan and M. Robin, On the convergence of the discrete time dynamic programming equation for general semigroups. SIAM J. Control Optim. 20(1982), No. 5, $722-746$.

2. I. I. Gikhman and A. V. Skorokhod, Introduction to the theory of random processes. (Russian) 2nd ed. Nauka, Moscow, 1977.

3. A. N. Shiryaev, Statistical sequential analysis. Optimal stopping rules. (Russian) 2nd ed. Nauka, Moscow, 1976.

(Received 7.03.2005; revised 12.09.2005)

Author's address:

A. Tsereteli Kutaisi State University

59, Tamar Mepe St., Kutaisi 4600

Georgia 